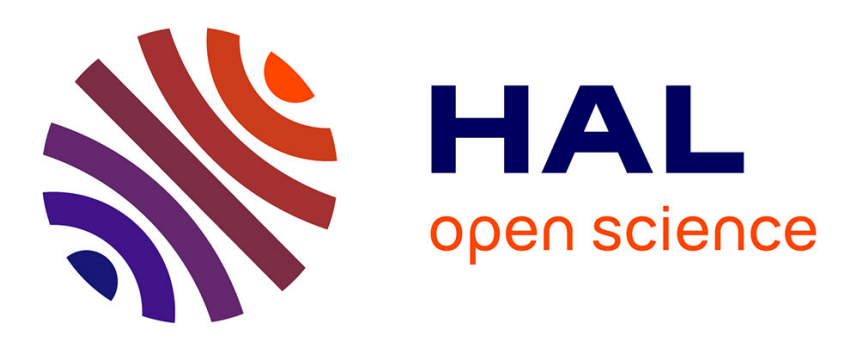

\title{
Effect of Planning Period on MPC-based Navigation for a Biped Robot in a Crowd
}

\author{
Matteo Ciocca, Pierre-Brice Wieber, Thierry Fraichard
}

\section{To cite this version:}

Matteo Ciocca, Pierre-Brice Wieber, Thierry Fraichard. Effect of Planning Period on MPC-based Navigation for a Biped Robot in a Crowd. IROS 2019 - IEEE/RSJ International Conference on Intelligent Robots and Systems, Nov 2019, Macau, China. pp.1-8, 10.1109/IROS40897.2019.8968070 . hal-02267426

\section{HAL Id: hal-02267426 \\ https://hal.inria.fr/hal-02267426}

Submitted on 19 Aug 2019

HAL is a multi-disciplinary open access archive for the deposit and dissemination of scientific research documents, whether they are published or not. The documents may come from teaching and research institutions in France or abroad, or from public or private research centers.
L'archive ouverte pluridisciplinaire HAL, est destinée au dépôt et à la diffusion de documents scientifiques de niveau recherche, publiés ou non, émanant des établissements d'enseignement et de recherche français ou étrangers, des laboratoires publics ou privés. 


\title{
Effect of Planning Period on MPC-based Navigation for a Biped Robot in a Crowd
}

\author{
Matteo Ciocca ${ }^{1}$, Pierre-Brice Wieber ${ }^{2}$, Thierry Fraichard ${ }^{1}$
}

\begin{abstract}
We control a biped robot moving in a crowd with a Model Predictive Control (MPC) scheme that generates stable walking motions, with automatic footstep placement. Most walking strategies propose to re-plan the walking motion to adapt to changing environments only once at every footstep. This is because a footstep is planted on the ground, it usually stays there at a constant position until the next footstep is initiated, what naturally constrains the capacity for the robot to react and adapt its motion in between footsteps. The objective of this paper is to measure if re-planning the walking motion more often than once at every footstep can lead to an improvement in collision avoidance when navigating in a crowd. Our result is that re-planning twice (or more) during each footstep leads to a significant reduction of the number of collisions when walking in a crowd, but depends on the density of the crowd.
\end{abstract}

\section{INTRODUCTION}

For a biped robot moving in a crowd, two things that should be avoided are to fall and to collide with people. Following the concept of Capturability [1], we can guarantee that the robot will always be able to stop in a few footsteps and maintain its balance forever. Following the concept of Inevitable Collision States [2], it is impossible to guarantee that collisions will never happen in a dynamic and uncertain environment. It is nonetheless possible to guarantee that the robot will be able to stop before a collision takes place, should this collision be inevitable. This property is called Passive Safety [3]. It has already been used effectively with self-driving cars [4], autonomous helicopters [5] and mobile robots in human environments [6].

Capturability has previously been used in Model Predictive Control (MPC) schemes that generate stable walking motions, with automatic footstep placement, to guarantee both fall avoidance and Passive Safety [7]. MPC is an iterative control scheme that operates as follows: at each discrete time instant $t_{i}$, a motion plan made up of a sequence of $N$ control actions is computed. The motion plan is valid from $t_{i}$ to $t_{i+N}$, where $N$ is the planning horizon. The robot then executes the first control action of the motion plan. The planning process is repeated at the next time instant $t_{i+1}$, until the goal is reached. Let $T$ denote the fixed time step (in seconds) between two consecutive time instants $\left[t_{i}, t_{i+1}\right]$. In MPC-based walking strategies such as [7], [8], [9], [10], [11]

This work has been partially supported by the LabEx PERSYVAL-Lab (ANR-11-LABX-0025-01), EU H2020 Comanoid Research and Innovation Action (RIA).

1 Univ. Grenoble Alpes, Inria, CNRS, Grenoble INP, LIG, 38000 Grenoble, France matteo.cioccadinria.fr, thierry.fraichardeinria.fr

2 Univ. Grenoble Alpes, Inria, 38000 Grenoble, France pierre-brice.wieberainria.fr or [12], this time step $T$ is usually small. In [7] for instance, $T$ is set to the duration of the double support phase of the walking cycle of the biped robot, i.e. 0.1[s]. This choice is primarily made to ensure the balance of the walking motions.

Once a footstep is planted on the ground, it usually stays there at a constant position until the next footstep is initiated. This naturally constrains the capacity for the robot to react and adapt its motion in between footsteps. As a result, the walking strategies in [13], [14], [15] propose to replan the walking motion to adapt to changing environments only when a new footstep is initiated. In contrast, the MPC scheme outlined above leads to re-planning the walking motion every $0.1[\mathrm{~s}]$. The objective of this paper is to measure if re-planning the walking motion more often than once at every footstep can lead to an improvement in collision avoidance when navigating in a crowd. As an element of comparison, it has been shown in [16] that when considering only the balance of a biped robot, reacting more often than every $0.2[\mathrm{~s}]$ to potential perturbations leads to no practical improvement in the maximal tracking error.

Outline of the Paper: Section II presents the global motion planning framework used herein. Then Section III details the walking/capturability and collision avoidance constraints specific to the biped walking case considered. The MPC framework for biped locomotion is outlined in Section IV. The crowd scenario that is used for our evaluation purposes is described in Section V-A whereas Section V-B presents the specific robot parameters used. The results of the collision rate obtained using simulated crowd scenarios are finally presented and discussed in Section VI.

\section{Motion Planning Through Optimization}

A motion is made up by a sequence of actions. Among possible motions, we want the biped robot to perform a walking motion that avoids falling. And, when a biped robot walks among people, it should perform a motion that also avoids collisions. In our case, such motion can be achieved by satisfying a closed convex set of constraints expressed as linear inequalities of the form:

$$
\underline{q} \leq G \mathbf{u} \leq \bar{q},
$$

where equality constraints can be expressed with $q=\bar{q}$, $\mathbf{u}=\left\{u_{1}, u_{2}, \cdots\right\}$ is a sequence of actions, $(q, \bar{q})$ and $G$ are vectors and matrix of proper dimension. In our case $\mathbf{u} \in \mathbb{R}^{2 N+2 m}$, where $N$ control inputs to robot's $2 \mathrm{D}$ system model, and $m$ future footstep positions in 2D plane. Once these constraints are satisfied, we choose the collision-free walking motion that minimizes the deviation from a given 
reference, e.g. robot maintains a constant walking speed. In our case, the reference deviation are expressed as a convex quadratic function:

$$
f(\mathbf{u})=\mathbf{u}^{T} Q_{r} \mathbf{u}+\mathbf{u}^{T} q_{r}
$$

where $q_{r}$ and $Q_{r}$ are a vector and matrix of proper dimension. Constraints and reference deviation are satisfied and minimized altogether, solving the following Quadratic Problem (QP):

$$
\begin{array}{ll}
\underset{\mathbf{u}}{\operatorname{minimize}} & f(\mathbf{u}) \\
\text { subject to } & \underline{q} \leq G \mathbf{u} \leq \bar{q} .
\end{array}
$$

This is a linearly constrained convex optimization problem, with a global optimum $\mathbf{u}^{*}$ [17]. Solving problem (3) provides a motion plan $\mathbf{u}$ for the robot to perform. We solve it with LexLS [18].

\section{Motion Constraints AND OBJECTIVES}

\section{A. Dynamics of Walking}

The horizontal motion of the Center of Mass (CoM) $c \in$ $\mathbb{R}^{2}$ of a humanoid robot is linearly related to the Center of Pressure $(\mathrm{CoP}) p \in \mathbb{R}^{2}$ of the contact forces when walking with a constant height on a flat ground:

$$
\ddot{c}=\omega^{2}(c-p)
$$

where $\omega^{2}=g / h, h$ is the height of the CoM above the ground and $g$ is the norm of the gravity vector. This assumes a zero rate of change of the centroidal angular momentum [19]. Since contact forces with the ground are unilateral, the CoP resides in the support polygon [19]:

$$
p-s_{j} \in \mathcal{P}
$$

where $s_{j} \in \mathbb{R}^{2}$ is the position of the $j^{\text {th }}$ footstep on the ground. The CoM position resides in a closed convex region due to the maximal leg length of the robot [20]:

$$
c-s_{j} \in \mathcal{L}
$$

Biped robots should not cross their legs while walking. Given two consecutive footstep positions $\left(s_{j}, s_{j+1}\right)$ and a region $\mathcal{S}_{j}$ where the legs do not cross, we enforce:

$$
s_{j+1}-s_{j} \in \mathcal{S}_{j}
$$

The robot can realize a stable walk by continuously satisfying these constraints into the future [10]:

$$
\text { Walking }(W) \equiv(5) \wedge(6) \wedge(7) \text {. }
$$

This set of constraints can be expressed as linear inequalities.

\section{B. Capturability Constraint}

The biped robot is said to be 0-step capturable [1] when it can maintain balance indefinitely, without having to make any footstep. We enforce this situation by introducing the following equality constraint [21]:

$$
\operatorname{Stop}(S) \equiv \dot{\xi}=0
$$

where $\xi \in \mathbb{R}^{2}$ is the Capture Point defined as:

$$
\xi=c+\omega^{-1} \dot{c}
$$

$(W)-(S)$ represent together a closed convex set.

\section{Collision Avoidance}

Consider a crowd of $Z$ persons. The robot avoids collisions with each person $z_{k}$ by keeping a minimal separation distance from its CoM:

$$
\left\|c-z_{k}\right\| \geq \sigma_{0}
$$

for all $k \in\{1, \ldots, Z\}$. In this equation, $z_{k} \in \mathbb{R}^{2}$ is the position of the $k^{t h}$ person and $\sigma_{0}$ is the minimal separation distance. In our implementation, this nonconvex constraint on collision avoidance $(C)$ is approximated with a separating line:

$$
\text { Collision Avoidance }(C) \equiv d_{k} \geq \sigma
$$

where

$$
d_{k}=\left(n_{k}\right)^{T}\left(c-z_{k}\right)
$$

$n_{k} \in \mathbb{R}^{2}$ is a unit normal vector that points from the center of the $k^{t h}$ person to the CoM of the robot. An advantage of this approach is that, by being conservative, this linear overapproximation is always safe with respect to the nonlinear problem.

\section{Robot's Perception}

Assume the biped robot is equipped with a range sensor, e.g. laser telemeter or range camera, and it can only perceive a subset of people composing the crowd. This subset is the robot's field of view, denoted FoV. Its shape is arbitrary and it depends on the current surrounding of the robot and the maximum range of its sensor. In our case, the FoV has a circular shape and it is the maximal distance around the robot $R_{\max }$ at which it is capable of perceiving persons/objects: $\left\|c-z_{k}\right\| \leq R_{\max }$. Occlusions in perception are disregarded, making the robot aware of the current position of everybody within the FoV.

\section{E. Walking References}

The robot should walk at a certain speed $\dot{c}_{r}$ and keep the CoP $p$ as close as possible to the center of the foot on the ground $s$, to improve the robustness of the robot against perturbations [19]. These are referred as:

$$
\text { References }(R) \equiv\left\{\begin{array}{l}
\dot{c}-\dot{c}_{r}=0 \\
p-s=0
\end{array}\right.
$$

and they can be expressed as a convex quadratic function [10]. 


\section{Model Predictive Control For Biped Walking}

Model Predictive Control (MPC) is used for biped locomotion with automatic footsteps placement [10]. Time is discretized in a sequence of time instants $t_{i}, i \in \mathbb{N}$. Each time interval $\left[t_{i}, t_{i+1}\right]$ has a fixed duration $T$ in seconds. Assuming the robot is walking on a flat ground, the motion of the CoM of a biped robot can be modeled in both horizontal coordinates as a linear discrete time system [21]:

$$
\hat{c}_{i+1}=A \hat{c}_{i}+B \dot{p}_{i}
$$

where

$$
\begin{aligned}
A & =\left[\begin{array}{ccc}
1 & \sinh (T \omega) / \omega & \cosh (T \omega) / \omega^{2}-1 / \omega^{2} \\
0 & \cosh (T \omega) & \sinh (T \omega) / \omega \\
0 & \sinh (T \omega) \omega & \cosh (T \omega)
\end{array}\right] \\
B & =\left[\begin{array}{c}
T-\sinh (T \omega) / \omega \\
1-\cosh (T \omega) \\
-\omega \sinh (T \omega)
\end{array}\right] .
\end{aligned}
$$

$\hat{c}_{i}=\left(c_{i}, \dot{c}_{i}, \ddot{c}_{i}\right)$ and $\hat{c}_{i+1}=\left(c_{i+1}, \dot{c}_{i+1}, \ddot{c}_{i+1}\right)$ are two consecutive states, and $\dot{p}_{i}$ is the velocity of the CoP, it is the control input of the system. A linear relationship is applied to the selection of the footsteps as:

$$
s_{i+1}=V_{i}^{c} s_{i}^{c}+V_{i}^{f} s_{i}^{f}
$$

where $s_{i}$ is the current footstep on the ground, $s_{f}$ is the future footstep position and $V_{i}^{c}$ and $V_{i}^{f}$ are cyclic timevarying scalars that determine which footstep is on the ground at what time (details are provided in [10]). A control action is therefore a couple of variables:

$$
u_{i}=\left[\begin{array}{ll}
\dot{p}_{i} & s_{i}^{f}
\end{array}\right] \text {. }
$$

MPC approach: At each time $t_{i}$, given the current state and footstep onto the ground $\left(\hat{c}_{i}, s_{i}^{c}\right)$, a single QP (3) is solved to plan a motion, composed by a sequence of $N$ control actions $\mathbf{u}^{N}=\left\{u_{1}, \cdots, u_{N}\right\}$. The couple $u_{j}$ represents: the control input $\dot{p}_{i+j-1}$ and $s_{i+j-1}^{f}$ footstep on the ground both at time instant $t_{i+j-1}$ and kept constant for a duration $T$, for $j=\{1,2, \cdots, N\}$. The motion is valid for $N$ time instants (from $t_{i}$ to $t_{i+N}$ ) and it has a duration $N T$, where $N$ is called the planning horizon. Usually, the first control action $u_{1}$ is executed, i.e. $\dot{p}_{i}$ is applied to the CoM dynamics (11) and the robot takes $s_{i}^{f}$ footstep (or keeps $s_{i}^{c}$ on the ground). Then at time $t_{i+1}$, the whole motion planning process is repeated.

We call the planning period $M$, with $M \in\{1,2, \cdots, N\}$, the number of time instants before the motion planning process is repeated. It corresponds to applying $M$ control actions before repeating the process at time $t_{i+M}$.

\section{A. Robot Navigation Scheme with Fall avoidance and Pas- sive Safety guarantees}

Fall avoidance for a biped robot is guaranteed if the robot can keep its balance indefinitely. Passive Safety (PS) guarantees that the robot is able to stop before a collision occurs: if a collision is inevitable, at least the robot will be at rest when that happens. PS can be proved using a conservative model of the surrounding environment [3]. Fall avoidance and PS are guaranteed together in a single MPC scheme for biped robots combining the conservative model that anticipates the motion of people (18) and the constraint $(S)$ to ensure that the robot can stop (keeping its balance indefinitely) before any collision happens [7].

The main idea is that at time instant $t_{i}$ the robot tries to compute a valid motion for $N$ time instants in which it will walk without colliding for few footsteps and additionally maintain balance in the last footstep forever, i.e. stop. If it cannot compute such motion, it can always execute all the remaining $N-M$ actions of the last computed motion at time instant $t_{i-M}$ as fallback. As a consequence, the robot walks without colliding and it will stop balancing on its last footstep on the ground (fall avoidance guaranteed) before any collision occurs (PS guaranteed). Before executing the remaining actions of the last computed motion as fallback, we go through a simple loop to find a motion valid for the largest number of time instants: $\{N-M, N-M+1, \cdots, N\}$, that satisfies all constraints.

\section{B. Newton method}

While the linear approximation in $(C)$ is safe with respect to the nonlinear problem (9), it over-constrains the behavior of the robot. To treat collision avoidance as a nonlinear problem we apply a Newton method [17] each time we solve the QP (3). By an abuse of notation, after each iteration $j$, the normal vectors $n_{j}$ corresponding to each person is updated with the previous optimal solution $\mathbf{u}_{j-1}^{*}$

$$
n_{j}=n_{j-1}\left(\mathbf{u}_{j-1}^{*}\right) \text {. }
$$

The iteration stops when:

$$
\left\|f\left(\mathbf{u}_{j-1}^{*}\right)-f\left(\mathbf{u}_{j}^{*}\right)\right\| \leq \epsilon .
$$

The maximum number of iterations is set to max Iter and $\epsilon$ is chosen arbitrarily small (Table I). Thanks to the safe linear approximation of $(C)$, feasible iterates are always generated. Note that the convergence condition (16) does not guarantee optimality. The main interest of this work is not to guarantee optimality of the motion with the Newton method, but to plan a motion that treat collision avoidance as close as possible to a nonlinear problem.

\begin{tabular}{ccc}
\hline Parameter & Symbol & Value \\
\hline Newton step Iterations & max_Iter & 5 \\
Convergence Parameter & $\epsilon$ & $10^{-4}$ \\
\hline
\end{tabular}

TABLE I: Newton method parameters.

\section{CROWD AND Robot Parameters}

\section{A. Crowd}

One possible model for the crowd behavior could be that every person is very careful in trying to avoid all collisions with the robot. With such a strong bias, the evaluation of the robot's own strategy would not be very meaningful [22]. 
Another extreme could be a crowd specifically aiming at collisions with the robot, which would once again corrupt the evaluation of the robot's strategy. One way to evaluate the robot's strategy and the robot's strategy alone, could be to consider that the robot is actually the only one in charge to avoid collisions. This leads us to considering a crowd not paying attention to the presence of the robot. We consider therefore people walking in a constant direction at constant speed. We consider the worst case scenario where the robot is walking in the opposite direction of the crowd.

The robot needs to avoid collisions in the present and into the future, we need therefore a model that anticipate the motion of people. In our crowd scenario: (i) people walk at a constant velocity and they do not try to avoid the robot and (ii) collisions among people are disregarded. The robot uses these information to anticipate the motion of people. We also consider uncertainties in position and velocity estimations of the crowd with respect to the real position $z_{k}$ and velocity $\dot{z}_{k}$ :

$$
\tilde{z}_{k}=z_{k}-\hat{z}_{k}, \tilde{\dot{z}}_{k}=\dot{z}_{k}-\hat{\dot{z}}_{k}
$$

and for collision avoidance $(C)$, we account for this uncertainty by adapting the minimal separation distance in a conservative way:

$$
\sigma(t)=\sigma_{0}+\left\|\tilde{z}_{k}\right\|+\left\|\tilde{\dot{z}}_{k}\right\| t .
$$

Here, given the magnitude of the uncertainty in position $\left\|\tilde{z}_{k}\right\|$, we consider that the real position $z_{k}$ lies in a circle of radius $\left\|\tilde{z}_{k}\right\|$ centered at the estimated position $\hat{z}_{k}$. Furthermore, given the magnitude of the uncertainty in velocity $\left\|\tilde{\dot{z}}_{k}\right\|$, the radius of this circle increases at a rate $\left\|\tilde{\dot{z}}_{k}\right\| t$ as it moves with the estimated velocity $\hat{\dot{z}}_{k}$.

We consider 100 different random crowds, composed of $Z$ people that differ in the initial positions $\left\{z_{k}^{x}, z_{k}^{y}\right\}$ and speeds $y$ : $\left\{\dot{z}_{k}^{y}\right\}$ of the participants. The initial positions of the people vary uniformly over an area of $10 \times 8\left[\mathrm{~m}^{2}\right]$. For all people the velocity along $x$ is chosen constant $0.5[\mathrm{~m} / \mathrm{s}]$, while for each person speed along $y$ is picked randomly in an interval

\begin{tabular}{|c|c|c|c|}
\hline Parameter & Symbol & Value & Unit \\
\hline Size of the crowd & $\mathrm{Z}$ & $\{8,16,32\}$ & ppl \\
\hline $\begin{array}{l}\text { Number of randomly } \\
\text { generated crowds (for size } Z \text { ) }\end{array}$ & - & 100 & - \\
\hline Speed of the crowd & $\begin{array}{l}\dot{z}_{k}^{x} \\
\dot{z}_{k}^{y}\end{array}$ & $\begin{array}{c}0.5 \\
{[-0.2,0.2]}\end{array}$ & $\begin{array}{l}\mathrm{m} / \mathrm{s} \\
\mathrm{m} / \mathrm{s}\end{array}$ \\
\hline min. separation distance (18) & $\sigma_{0}$ & 1 & $\mathrm{~m}$ \\
\hline Uncertainty (18) & $\begin{array}{l}\left\|\tilde{z}_{k}\right\| \\
\left\|\tilde{\dot{z}}_{k}\right\|\end{array}$ & $\begin{array}{l}\{0,0.15,0.30\} \\
\{0,0.05,0.10\}\end{array}$ & $\begin{array}{c}\mathrm{m} \\
\mathrm{m} / \mathrm{s}\end{array}$ \\
\hline
\end{tabular}
$[-0.2,0.2][\mathrm{m} / \mathrm{s}]$.

TABLE II: Crowd Parameters.

\section{B. Robot}

The parameters of the simulated robot were selected according HRP-2 robot [23]. In our MPC framework, the walking cycle of the biped robot is divided in two stages: single and double support phases (SS and DS). SS duration is $0.7[\mathrm{~s}]$ and DS duration is $0.1[\mathrm{~s}]$, resulting in a step cycle of $s_{d}=0.8[\mathrm{~s} /$ footstep], and the time step $T$ is equal to the DS duration $(T=0.1[\mathrm{~s}])$. These duration have been used to generate stable walking motions [9]: changing the duration of SS, DS (and hence $T$ ) is out of the scope of this work. We control the robot with the MPC scheme in Section IV-A that guarantees fall avoidance and PS.

When the robot walks against a crowd, the robot perceives people around it within a FoV based on a specific sensor, e.g. laser scanner. The choice of FoV is not treated fully as in [3], but it is chosen greater than a certain lower bound in order to guarantee PS [7]. This lower bound depends on plan duration $N T$ and, robot's and people walking speed: $R_{\max } \geq N T\left(\dot{z}^{x}+\dot{c}_{r}^{x}\right)$.

With the MPC scheme in Section IV-A, the robot can plan up to $\left\lceil\left(N T / s_{d}\right)\right\rceil$ footsteps ahead. We choose to plan up to two full footsteps ahead, $N=16$, since it is a standard choice for biped locomotion with our MPC scheme [21], [10]. In our case, with $M=1$, the re-planning takes place 8 times within each footstep (re-plans every $0.1[\mathrm{~s}]$ ). We consider the case where the robot re-plans 2 times within each footstep: at every footstep and at the midpoint of each footstep $M=4$ (re-plans every $0.4[\mathrm{~s}]$ ), and the case where the robot re-plans 1 time between each footstep: only at every footstep $M=8$ (re-plans every $0.8[\mathrm{~s}]$ ).

\begin{tabular}{cccc}
\hline Parameter & Symbol & Value & Unit \\
\hline Height of CoM (4) & $h$ & 0.80 & $\mathrm{~m}$ \\
Min. Feet & $f_{s}$ & 0.07 & $\mathrm{~m}$ \\
Separation (7) & $f$ & $(0.24,0.14)$ & $(\mathrm{m}, \mathrm{m})$ \\
Feet Dimensions (5) & $l$ & $(0.24,0.30)$ & $\mathrm{m}$ \\
Leg Stride (6) & $s_{d}$ & 0.80 & s/footstep \\
Step Cycle & $N$ & 16 & - \\
Planning Horizon & $\left\lceil\left(N T / s_{d}\right)\right\rceil$ & 2 & footstep \\
future footstep & $T$ & 0.10 & $\mathrm{~s}$ \\
positions & $M$ & $\{1,4,8\}$ & - \\
Time Step & $(0.5,0.0)$ & $\mathrm{m} / \mathrm{s}$ \\
Planning Period & $\left.\dot{c}_{r}^{x}, \dot{c}_{r}^{y}\right)$ & 4 & $\mathrm{~m}$ \\
Reference Speed $(R)$ & & & \\
FoV distance & $R_{\max }$ & &
\end{tabular}

TABLE III: Robot Parameters.

\section{EfFect of Planning Period on Collision Rate}

The effect of $M$ on the collision rate for a biped robot traversing a moving crowd is evaluated numerically, as seen in Fig. 1. The initial positions of the people are randomly chosen in front of the robot outside the FoV. The crowd parameters are summarized in Table II, the robot parameter in Table III.

\section{A. Choice of $M$ for Crowd Density}

For each uncertainty $\left(\left\|\tilde{z}_{k}\right\|,\left\|\tilde{\dot{z}}_{k}\right\|\right)$, we simulate 100 crowds of $Z$ people. Each simulation lasts for 20 [s], or until a collision occurs. Table IV shows the performance of collision avoidance by counting the total number of collisions for each choice of planning period $M$ and crowds size $Z$ with varying uncertainty $\left(\left\|\tilde{z}_{k}\right\|,\left\|\tilde{\dot{z}}_{k}\right\|\right)$. We count the number of collisions for each combination $\{M, Z\}$ that happened in 900 simulations. 
The results indicates that re-planning twice (or more) during each footstep leads to a significant reduction of the number of collisions when walking in a crowd. But this reduction depends on the density of the crowd.

When the robot is traversing few people, $Z=8$, the choice of $M=8$ performed worse than $M=4$ by $77 \%$, and $M=4$ performed worse than $M=1$ by $38 \%$. For low-density crowds, collisions when re-planning 8 times every footstep $(M=1)$ are less than twice compared to re-plan once every footstep $(M=8)$ and less than twice the collisions compared to re-plan twice every footstep $(M=4)$. In this case replanning less often to save computational power comes at the expense of a decay in collision avoidance performance, therefore we favor the planning period $M=1$.

When a biped robot is traversing many people, $Z=\{16,32\}$, the choice of $M=8$ performed worse than $M=4$ by $53 \%$, and $M=4$ performed worse than $M=1$ by $20 \%$. For high-density crowds, collisions when re-planning 8 times every footstep $(M=1)$ are more than twice the collisions compared to re-plan once every footstep ( $M=8)$ but only reduced them by a fifth compared to replan twice every footstep $(M=4)$. We can then favor a planning period $M=4$ over $M=1$ (re-planning 4 times less) to save computational power, since it performs worse by only a fifth in collision avoidance.

\section{B. Collisions and Uncertainty}

We now give some insight on the relationship between collisions and uncertainty. We summarize in Table $\mathrm{V}$ the total number of collisions occurring in 300 simulations with different crowd sizes $Z$, for each planning period $M$ and various amount of uncertainty $\left(\left\|\tilde{z}_{k}\right\|,\left\|\tilde{\dot{z}}_{k}\right\|\right)$.

When there is uncertainty, the robot accounts for it by being more cautious: uncertainty is represented as an additional area where people might be into the future and the robot needs to avoid it.

As result, the number of collisions decreases in the presence of uncertainty. We can observe that uncertainty on speed has a higher impact on the number of collisions than uncertainty on position.

\begin{tabular}{cccc} 
& \multicolumn{3}{c}{ Collisions } \\
\cline { 2 - 4 } Crowd Size $Z[\mathrm{ppl}]$ & $M=8$ & $M=4$ & $M=1$ \\
\hline 8 & 324 & 74 & 46 \\
16 & 557 & 289 & 235 \\
32 & 638 & 357 & 296 \\
\hline total collisions & 1539 & 720 & 577 \\
\hline
\end{tabular}

TABLE IV: Collision Rate of $M$ for crowd density.

\section{CONCLUSIONS}

We control a biped robot moving in a crowd with our MPC framework that guarantee fall avoidance and PS [7]. Most walking strategies propose to re-plan the walking motion to adapt to changing environments only once at every footstep. This is because a footstep is planted on the ground, it usually stays there at a constant position until the next footstep is

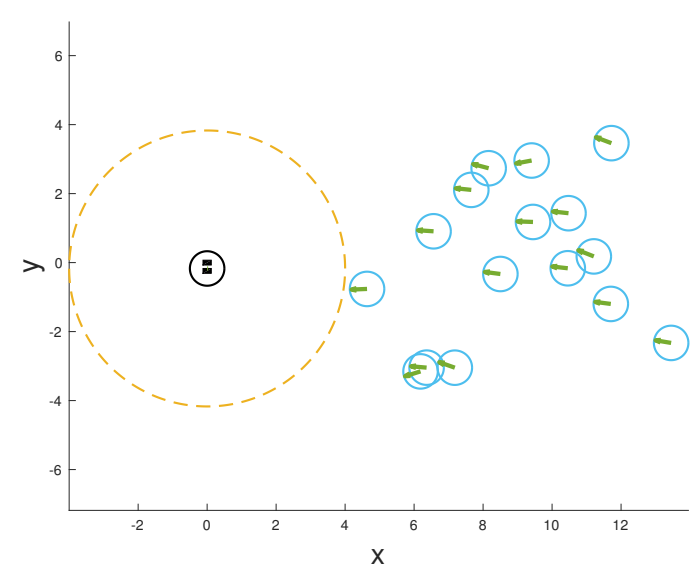

Fig. 1: $t_{0}$ in a crowd scenario with $Z=16$ : robot in black, people in blue (collisions among people are disregarded), the FoV is the dashed circle and the instantaneous velocity of each person and robot is represented by the green vector. The robot is asked to walk to the right with $\dot{c}_{r}^{x}=0.5[\mathrm{~m} / \mathrm{s}]$.

\begin{tabular}{lccccc}
\hline \multicolumn{2}{c}{ Uncertainty } & & \multicolumn{3}{c}{ Collisions } \\
\cline { 1 - 2 } \cline { 5 - 6 }$\left\|\tilde{\dot{z}}_{k}\right\|[\mathrm{m} / \mathrm{s}]$ & $\left\|\tilde{z}_{k}\right\|[\mathrm{m}]$ & & $M=8$ & $M=4$ & $M=1$ \\
\hline \multirow{2}{*}{0.00} & 0.00 & & 219 & 91 & 80 \\
& 0.15 & & 203 & 82 & 70 \\
& 0.30 & & 199 & 100 & 77 \\
\hline \multirow{2}{*}{0.05} & 0.00 & & 163 & 78 & 63 \\
& 0.15 & & 169 & 79 & 62 \\
& 0.30 & & 151 & 78 & 62 \\
\hline \multirow{2}{*}{0.10} & 0.00 & & 138 & 69 & 52 \\
& 0.15 & & 141 & 72 & 57 \\
& 0.30 & & 136 & 71 & 54 \\
\hline
\end{tabular}

TABLE V: Relationship between collisions and uncertainty.

initiated, what naturally constrains the capacity for the robot to react and adapt its motion in between footsteps. Our result shows that re-planning twice (or more) during each footstep, instead of only once, leads to a significant reduction of the number of collisions when walking in a crowd. In addition, the choice of re-planning can be done according to the density of the crowd. For highly-dense crowd, replanning more than twice during each footstep does not improve significantly the number of collisions.

As future work, we want to apply different synchronization for the re-planning. For example, the re-plan only at every footstep is synchronized with the DS phase of the robot. We want to synchronize this re-planning choice with the SS phase. And we would like to explore how the choice of replanning affects collision avoidance performance for a fixed crowd density with different crowd speeds.

\section{REFERENCES}

[1] T. Koolen, T. de Boer, J. Rebula, A. Goswami, and J. Pratt, "Capturability-based analysis and control of legged locomotion, part 1: Theory and application to three simple gait models," The International Journal of Robotics Research, vol. 31, no. 9, pp. 1094-1113, aug 2012. 
[2] T. Fraichard and H. Asama, "Inevitable collision states. a step towards safer robots?" Advanced Robotics, vol. 18, no. 10, pp. 1001-1024, 2004, selected from 2003 IEEE-RSJ Int. Conf. on Intelligent Robots and Systems (IROS).

[3] S. Bouraine, T. Fraichard, and H. Salhi, "Provably safe navigation for mobile robots with limited field-of-views in dynamic environments," Autonomous Robots, vol. 32, no. 3, pp. 267-283, nov 2011.

[4] K. Macek, D. A. Vasquez Govea, T. Fraichard, and R. Y. Siegwart, "Towards Safe Vehicle Navigation in Dynamic Urban Scenarios," Automatika, 2009.

[5] S. Choudhury, S. Arora, and S. Scherer, "The planner ensemble and trajectory executive: A high performance motion planning system with guaranteed safety," in AHS 70th Annual Forum, Montral, Qubec, Canada, May 2014.

[6] S. B. Liu, H. Roehm, C. Heinzemann, I. Ltkebohle, J. Oehlerking, and M. Althoff, "Provably safe motion of mobile robots in human environments," in 2017 IEEE/RSJ International Conference on Intelligent Robots and Systems (IROS), Sept 2017, pp. 1351-1357.

[7] N. Bohorquez, A. Sherikov, D. Dimitrov, and P.-B. Wieber, "Safe navigation strategies for a biped robot walking in a crowd," in 2016 IEEE-RAS 16th International Conference on Humanoid Robots (Humanoids). IEEE, nov 2016.

[8] S. Kajita, F. Kanehiro, K. Kaneko, K. Fujiwara, K. Harada, K. Yokoi, and H. Hirukawa, "Biped walking pattern generation by using preview control of zero-moment point," in 2003 IEEE International Conference on Robotics and Automation (Cat. No.03CH37422), vol. 2, Sep. 2003, pp. $1620-1626$ vol.2.

[9] C. Dune, A. Herdt, E. Marchand, O. Stasse, P.-B. Wieber, and E. Yoshida, "Vision based control for Humanoid robots," in IROS Workshop on Visual Control of Mobile Robots (ViCoMoR), San Francisco, USA, United States, Sep. 2011, pp. 19-26.

[10] A. Herdt, H. Diedam, P.-B. Wieber, D. Dimitrov, K. Mombaur, and M. Diehl, "Online walking motion generation with automatic footstep placement," Advanced Robotics, vol. 24, no. 5-6, pp. 719-737, jan 2010.

[11] N. Scianca, M. Cognetti, D. De Simone, L. Lanari, and G. Oriolo, "Intrinsically stable mpc for humanoid gait generation," in 2016 IEEE-RAS 16th International Conference on Humanoid Robots (Humanoids), Nov 2016, pp. 601-606.

[22] T. Fraichard, "A Short Report about Motion Safety," 2006.
[12] M. Naveau, M. Kudruss, O. Stasse, C. Kirches, K. Mombaur, an P. Soures, "A reactive walking pattern generator based on nonlinear model predictive control," IEEE Robotics and Automation Letters, vol. 2, no. 1, pp. 10-17, Jan 2017.

[13] J. Chestnutt, M. Lau, G. Cheung, J. Kuffner, J. Hodgins, and T. Kanade, "Footstep planning for the honda ASIMO humanoid," in Proceedings of the 2005 IEEE International Conference on Robotics and Automation. IEEE, 2005.

[14] P. Karkowski and M. Bennewitz, "Real-time footstep planning using a geometric approach," in 2016 IEEE International Conference on Robotics and Automation (ICRA), May 2016, pp. 1782-1787.

[15] J. Garimort, A. Hornung, and M. Bennewitz, "Humanoid navigation with dynamic footstep plans," in 2011 IEEE International Conference on Robotics and Automation, 2011, pp. 3982-3987.

[16] N. A. Villa, J. Englsberger, and P.-B. Wieber, "Sensitivity of Legged Balance Control to Uncertainties and Sampling Period," in IROS 2019 - IEEE/RSJ International Conference on Intelligent Robots and Systems. MACAO, China: IEEE, Nov. 2019. [Online]. Available: https://hal.archives-ouvertes.fr/hal-02168523

[17] J. Nocedal and S. Wright, Numerical Optimization, ser. Springer Series in Operations Research and Financial Engineering. Springer New York, 2006.

[18] D. Dimitrov, A. Sherikov, and P.-B. Wieber, "Efficient resolution of potentially conflicting linear constraints in robotics," Aug. 2015 [Online]. Available: https://hal.inria.fr/hal-01183003

[19] P.-B. Wieber, R. Tedrake, and S. Kuindersma, "Modeling and control of legged robots," in Springer Handbook of Robotics. Springer, 2016 , pp. 1203-1234.

[20] C. Brasseur, A. Sherikov, C. Collette, D. Dimitrov, and P.-B. Wieber, "A robust linear MPC approach to online generation of $3 \mathrm{~d}$ biped walking motion," in 2015 IEEE-RAS 15th International Conference on Humanoid Robots (Humanoids). IEEE, nov 2015.

[21] A. Sherikov, "Balance preservation and task prioritization in whole body motion control of humanoid robots," Ph.D. dissertation, Grenoble Alpes, 2016.

[23] K. Kaneko, F. Kanehiro, S. Kajita, H. Hirukawa, T. Kawasaki, M. Hirata, K. Akachi, and T. Isozumi, "Humanoid robot hrp-2," in IEEE International Conference on Robotics and Automation, 2004 Proceedings. ICRA '04. 2004, vol. 2, April 2004, pp. 1083-1090 Vol.2. 